\title{
Static properties of small Josephson tunnel junctions in a transverse magnetic field
}

\author{
Monaco, R.; Aarøe, Morten; Mygind, Jesper; Koshelets, V.
}

Published in:

Journal of Applied Physics

Link to article, DOI:

$10.1063 / 1.2956711$

Publication date:

2008

Document Version

Publisher's PDF, also known as Version of record

Link back to DTU Orbit

Citation (APA):

Monaco, R., Aarøe, M., Mygind, J., \& Koshelets, V. (2008). Static properties of small Josephson tunnel junctions in a transverse magnetic field. Journal of Applied Physics, 104(2), 023906. https://doi.org/10.1063/1.2956711

\section{General rights}

Copyright and moral rights for the publications made accessible in the public portal are retained by the authors and/or other copyright owners and it is a condition of accessing publications that users recognise and abide by the legal requirements associated with these rights.

- Users may download and print one copy of any publication from the public portal for the purpose of private study or research.

- You may not further distribute the material or use it for any profit-making activity or commercial gain

- You may freely distribute the URL identifying the publication in the public portal

If you believe that this document breaches copyright please contact us providing details, and we will remove access to the work immediately and investigate your claim. 


\title{
Static properties of small Josephson tunnel junctions in a transverse magnetic field
}

\author{
R. Monaco, ${ }^{1, a)}$ M. Aaroe, ${ }^{2, b)}$ J. Mygind, ${ }^{2, c)}$ and V. P. Koshelets ${ }^{3, d)}$ \\ ${ }^{1}$ Istituto di Cibernetica del C.N.R., 80078, Pozzuoli, Italy \\ and Unita' INFM-Dipartimento di Fisica, Universita' di Salerno, 84081 Baronissi, Italy \\ ${ }^{2}$ Department of Physics, B309, Technical University of Denmark, DK-2800 Lyngby, Denmark \\ ${ }^{3}$ Institute of Radio Engineering and Electronics, Russian Academy of Science, Mokhovaya 11, Bldg. 7, \\ 125009, Moscow, Russia
}

(Received 8 April 2008; accepted 13 May 2008; published online 21 July 2008)

\begin{abstract}
The magnetic field distribution in the barrier of small planar Josephson tunnel junctions is numerically simulated in the case when an external magnetic field is applied perpendicular to the barrier plane. The simulations allow for heuristic analytical solutions for the Josephson static phase profile from which the dependence of the maximum Josephson current on the applied field amplitude is derived. The most common geometrical configurations are considered and, when possible, the theoretical findings are compared with the experimental data. () 2008 American Institute of Physics. [DOI: 10.1063/1.2956711]
\end{abstract}

The static (and dynamic) properties of a planar Josephson tunnel junction (JTJ) are well understood when an external magnetic field is uniformly applied in the junction plane. ${ }^{1}$ On the contrary, very little is known when a uniform magnetic field is applied perpendicularly to the barrier plane. The main reason why, since the discovery of the Josephson effect in 1962, only few papers have dealt with a transverse magnetic field ${ }^{2-4}$ is because demagnetization effects imposed by the electrodes geometry are awkward to take into account. In a recent paper $^{5}$ we provided an experimental proof that a transverse magnetic field can be much more capable than an in-plane one to modulate the critical current $I_{c}$ of a planar JTJ with proper barrier and electrodes geometry requirements. It is also possible to design the JTJ electrode geometry in such a way that it is totally insensitive to a transverse field. The possibility to have on the same chip JTJs having different sensitivities to an externally applied field can be very attractive in practical applications.

In this paper we push our analysis further by resorting to numerical magnetostatic simulations to find the field distribution $\mathbf{H}$ in the barrier plane of those JTJs having the most common rectangular and annular geometries. Once $\mathbf{H}$ is found empirically, the Josephson phase $\phi$, which is the difference between the complex wave function phases in the electrodes, can be obtained from the Josephson equation ${ }^{6}$

$$
\boldsymbol{\nabla} \phi=\frac{2 \pi d_{e} \mu_{0}}{\Phi_{0}} \mathbf{H} \times \mathbf{n}
$$

where $\mathbf{n}$ is a unit vector normal to the insulating barrier separating the two superconducting electrodes, $\mu_{0}$ is the vacuum permeability, and $\Phi_{0}=h / 2 e$ is the magnetic flux quantum. If the two superconducting films have thicknesses

\footnotetext{
${ }^{\text {a) }}$ Author to whom correspondence should be addressed. Electronic address roberto@sa.infn.it.

${ }^{b)}$ Electronic address: aaroe@ @ysik.dtu.dk.

${ }^{c)}$ Electronic address: myg@fysik.dtu.dk.

${ }^{\mathrm{d})}$ Electronic address: valery@ hitech.cplire.ru.
}

$d_{1,2}$ and London penetration depths $\lambda_{L 1,2}$ and $t_{j}$ is the barrier thickness, then the effective magnetic penetration $d_{e}$ is given by $^{7}$

$$
d_{e}=t_{j}+\lambda_{L 1} \tanh \frac{d_{1}}{2 \lambda_{L 1}}+\lambda_{L 2} \tanh \frac{d_{2}}{2 \lambda_{L 2}},
$$

which, in the case of thick superconducting films $\left(d_{i}>\right.$ $>\lambda_{L i}$ ), reduces to $d_{e} \approx \lambda_{L 1}+\lambda_{L 2}$ (being always $d_{i}>>t_{j}$ ).

In Cartesian coordinates, assuming that the tunnel barrier lies in the $x-y$ plan, Eq. (1) reduces to

$$
\frac{\partial \phi(x, y)}{\partial x} \propto-H_{y}, \quad \frac{\partial \phi(x, y)}{\partial y} \propto H_{x} .
$$

A planar JTJ is said to be electrically small when its dimensions are smaller than the Josephson penetration depth $\lambda_{J}$ $=\sqrt{\hbar / 2 e \mu_{0} d_{e} J_{c}}$, where $J_{c}$ is the Josephson current density that we assume to be uniform over the barrier area. For such JTJs, the self-induced field associated with the bias current can be neglected and the Josephson phase must satisfy the two-dimensional Laplacian equation ${ }^{6}$

$$
\frac{\partial^{2} \phi}{\partial x^{2}}+\frac{\partial^{2} \phi}{\partial y^{2}}=0
$$

with proper boundary conditions related to the value of the magnetic field components $H_{x}$ and $H_{y}$ on the junction perimeter. It was first pointed out in 1975 (Ref. 3) that in a transverse applied field $\mathbf{H}=H_{\perp} \hat{\mathbf{z}}$, the in-plane components $H_{x}$ and $H_{y}$ are ascribed to surface demagnetizing currents $\mathbf{j}_{\mathbf{s}}=\hat{\mathbf{z}} \times \mathbf{H}$ feeding the interior of the junction. Since these currents mainly flow on the film edges, the largest sensitivity to a transverse field occurs when the junction is formed at the film edges. On the contrary, if the barrier is placed well inside the superconducting films, the effect of a transverse field vanishes. Our task consists of numerically evaluating the field line distribution in the barrier plane, from which we determine an empirical analytical expression $\phi(x, y)$ for the phase profile which satisfies Eq. (3). Such a phase profile 
will allow the computation of the transverse magnetic diffraction pattern $I_{c}\left(H_{\perp}\right)$ for small JTJs having different geometries and to compare it with experimental data, if available. This is achieved by recalling that the maximum Josephson current is

$$
I_{c}=I_{0} \sqrt{\langle\sin \phi\rangle^{2}+\langle\cos \phi\rangle^{2}},
$$

in which the brackets \langle\rangle denote spatial averages over the junction area. Throughout the paper we assume that the applied transverse field is everywhere much smaller than the (lower) critical field $H_{C 1}$ which would force the films into the intermediate or normal state, i.e., that the superconductors are always in the flux-free Meissner regime.

\section{MAGNETOSTATIC SIMULATIONS}

In general, magnetostatic problems are based on the magnetic vector potential. However, where no electrical currents are present, the problem can be conveniently solved using the scalar magnetic potential. In fact, in a current free region $\boldsymbol{\nabla} \times \mathbf{H}=0$ allows the introduction of a scalar potential $V_{m}$ such that $\mathbf{H}=-\nabla V_{m}$. Using the constitutive relation $\mathbf{B}$ $=\mu_{0} \mu_{r} \mathbf{H}$, we can rewrite Maxwell's equation $\boldsymbol{\nabla} \cdot \mathbf{B}=0$ in terms of $V_{m}$

$$
-\nabla \cdot\left(\mu_{0} \mu_{\mathbf{r}} \nabla V_{m}\right)=0,
$$

in which the magnetic relative permittivity $\mu_{r}$ is spatially dependent. We assumed that the superconducting electrodes are thicker than their London penetration depths $\left(d_{i}>\right.$ $\left.>\lambda_{L i}\right)$, so that the London equation reduces to $\mathbf{B}=0$ everywhere inside the superconductors, i.e., $\mu_{r}=0$ (perfect diamagnetism) and the normal component of the magnetic flux density vanishes at the boundary $(\mathbf{n} \cdot \mathbf{B}=0)$. In the opposite limit, the films would become transparent to the transverse field and, in turn, the junction would lose its sensitivity to the transverse field. A uniform applied magnetic field $\mathbf{H}=H_{\perp} \hat{\mathbf{z}}$ is taken into account by imposing that sufficiently far away from the junction is $V_{m}=-H_{\perp} z$. All the simulations presented in this paper were carried out setting $H_{\perp}=1 \mathrm{~A} / \mathrm{m}$.

As a consequence of the definitions of Eq. (2), it is straightforward to show that Eq. (3) requires that $\partial^{2} V_{m} / \partial x \partial y=\partial^{2} V_{m} / \partial y \partial x$. Further, more importantly, we have

$$
\phi(x, y) \propto \int d x \frac{\partial V_{m}}{\partial y}=-\int d y \frac{\partial V_{m}}{\partial x} .
$$

The numerical solution of Eq. (5) was implemented in the COMSOL Multiphysics 3D Electromagnetics module for JTJs having different rectangular and annular geometries. Models with large geometric scale variations are always problematic to mesh, in particular if they contain thin layers with large aspect ratio. Therefore, one caveat of our modeling is that, in order to keep the number of mesh elements within the PC memory handling capability, the separation between the superconducting films, i.e., the tunnel barrier thickness $t_{j}$, could not be set to realistic values for a Josephson tunnel barrier $O(1 \mathrm{~nm})$. Our numerical modeling was tested against the magnetic field distribution around a superconducting disk (with radius $R$ larger than its thickness $d$ ) in the plane $z=0$, centered on the $z$ axis and immersed in a field

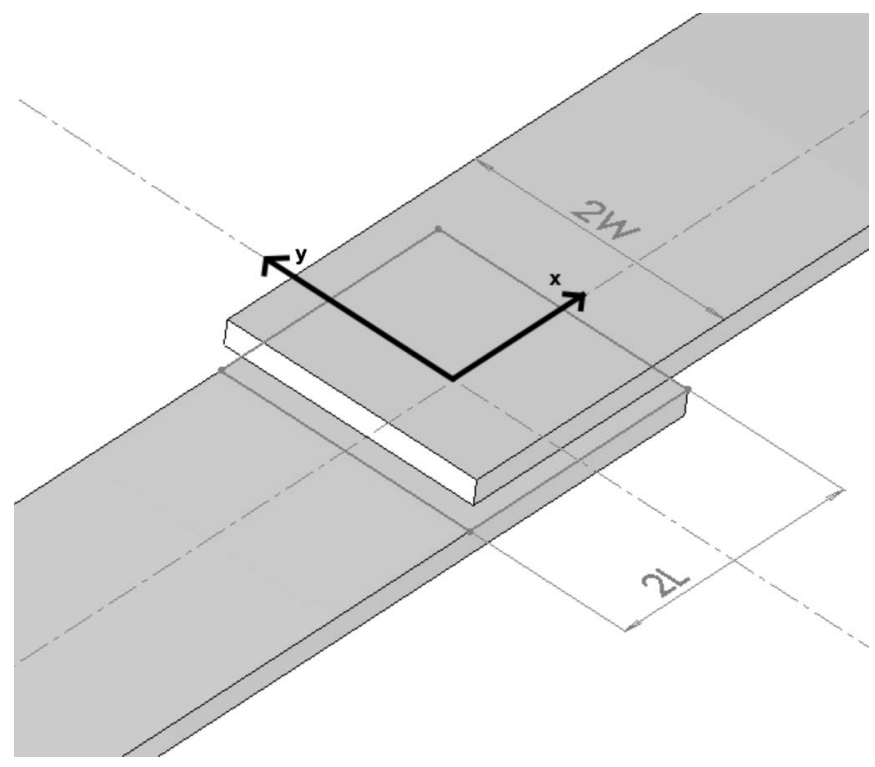

FIG. 1. Sketch of a overlap geometry junction. The center of the junction coincides with origin of our coordinate system.

$\mathbf{H}=H_{\perp} \hat{\mathbf{z}}$. More precisely, the radial dependence of the tangential field $H_{t}$ on the disk surface followed to a high accuracy the well-known expression ${ }^{8} H_{t}(r) \propto H_{\perp} r / \sqrt{R^{2}-r^{2}}$ everywhere except at the disk border, where the inverse square root singularity was replaced by a finite value $\hat{H}$ proportional to the square root of the disk aspect ratio, i.e., $\hat{H}=H_{\perp} \sqrt{R / d} .{ }^{9}$ This example is indicative of the fact that, in general, the magnetostatic response of any superconducting film structure is markedly dependent on the film aspect ratio. Furthermore, the Meissner state condition $\hat{H} \leq H_{C 1}$ allow us to estimate that for a $\mathrm{Nb}$ film with aspect ratio $O\left(10^{3}\right)$, the first flux penetration occurs when the applied transverse field $H_{\perp}$ is of the order of $10^{3} \mathrm{~A} / \mathrm{m}$.

\section{RECTANGULAR JUNCTIONS}

\section{A. Overlap-type junctions}

We begin our analysis with a JTJ obtained by the superposition of the extremities of two long and narrow parallel superconducting electrodes with equal widths. This so-called overlap geometry is depicted in Fig. 1 for a square junction, i.e., $W=L$. The tunnel barrier lies in the $z=0$ plane and its center coincides with the axis origin. Further, it has a length $2 L$ along the $x$-direction and a width $2 W$ along the $y$-direction. In the simulations the electrodes have a thickness $d=10 \mu \mathrm{m}$ and are $t_{j}=1 \mu \mathrm{m}$ apart. Such unrealistic barrier thickness only leads to an underestimation or the real amplitudes of the magnetic field between the junction electrodes. The film width $2 W$ and the overlapping length $2 L$ were varied in order to treat barriers with different aspect ratios $\beta=L / W$. Figures 2(a)-2(c) show the numerically obtained $V_{m}$ solutions in the barrier area of three overlap junctions having the same width $2 W=80 \mu \mathrm{m}$, but different lengths $2 L=20,80$, and $320 \mu \mathrm{m}$. By analyzing the properties of such plots we aim to infer an empirical, physically acceptable analytical form for $V_{m}(x, y, z=0)$. We observe that for any value of $\beta$, the scalar potential in the barrier is sym- 

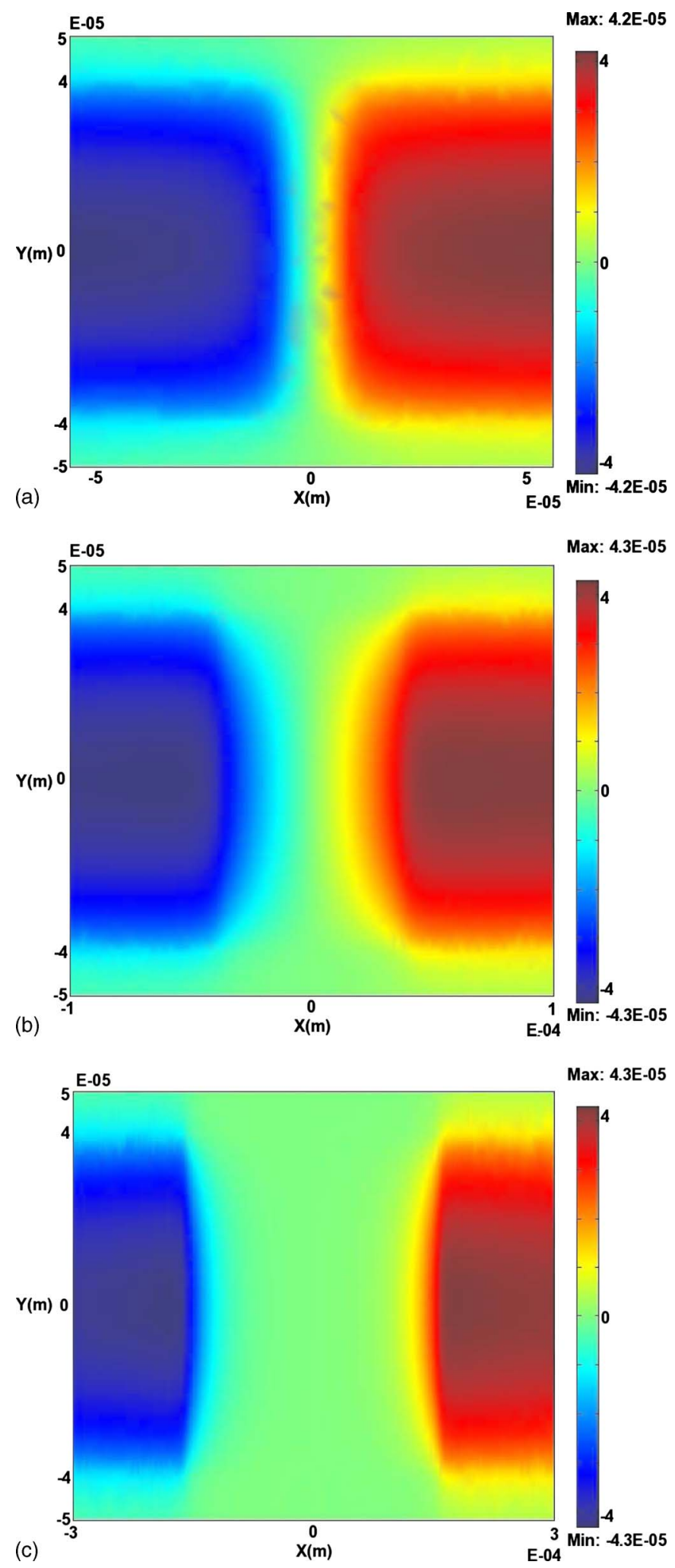

FIG. 2. (Color online) Numerically obtained magnetic scalar potential $V_{m}$ (in A) for three overlap planar Josephson tunnel junctions having the same width $2 W=80 \mu \mathrm{m}$, but different lengths: (a) $2 L=20 \mu \mathrm{m}(\beta=0.25)$, (b) $2 L=80 \mu \mathrm{m}(\beta=1)$, and (c) $2 L=320 \mu \mathrm{m}(\beta=4)$. The transverse externally applied magnetic field is $H_{\perp}=1 \mathrm{~A} / \mathrm{m}$.

metric with respect to the $x$-axis and antisymmetric with respect to the $y$-axis. In other words, the expression $V_{m}(x, y)$ we are looking for has to be an odd function of $x$ and an even function of $y$. Furthermore, we note that the potential decays
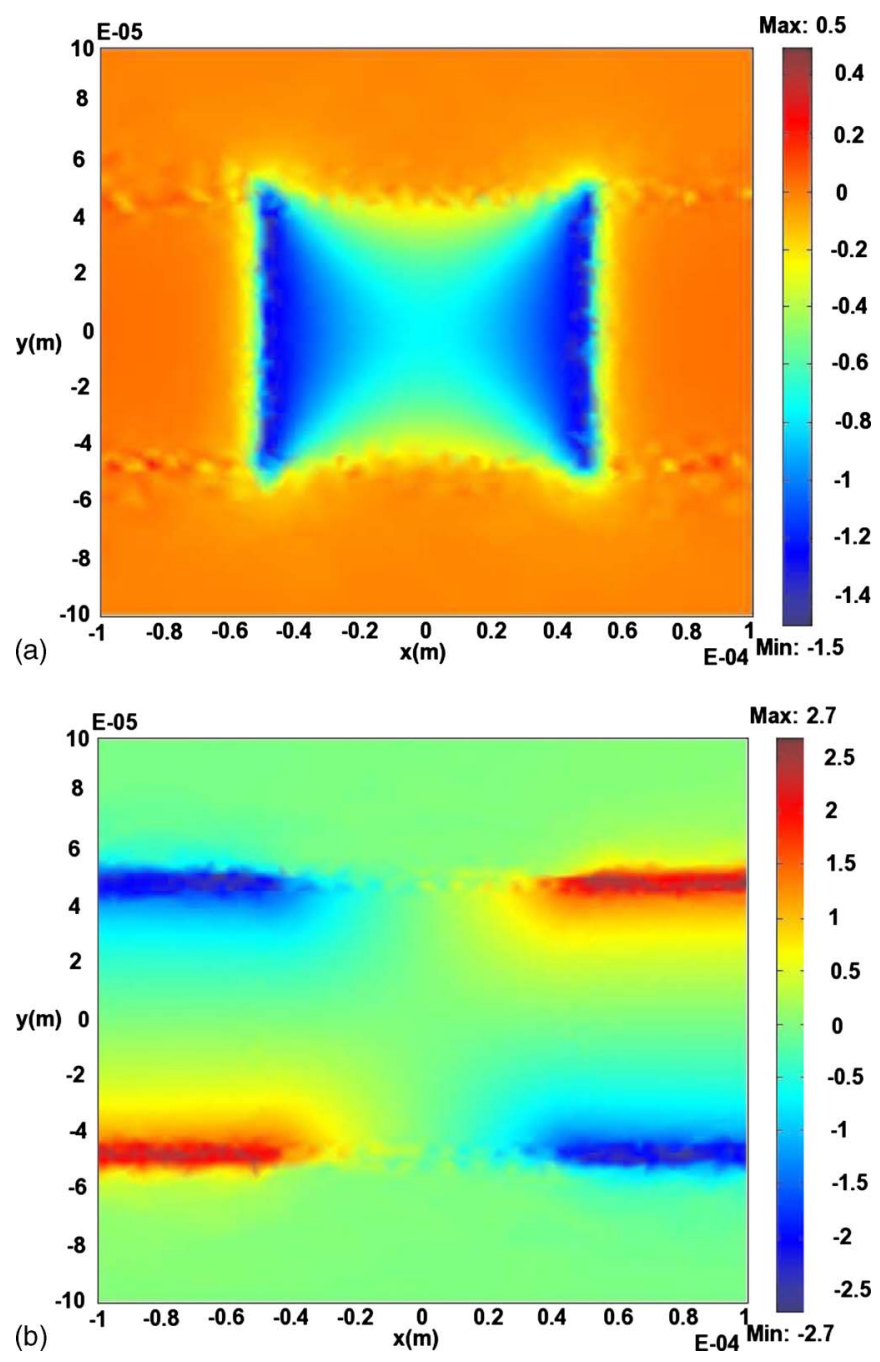

FIG. 3. (Color online) Numerically obtained in-plane magnetic field components in $\mathrm{A} / \mathrm{m}$ for a square overlap junction having $2 W=2 L=100 \mu \mathrm{m}$ in a transverse externally applied field $H_{\perp}=1 \mathrm{~A} / \mathrm{m}$. Color plots for (a) $H_{x}(x, y, z=0)$ and (b) $H_{y}(x, y, z=0)$.

from the junction corners over a distance $W$, being mostly null when $L>W$ (or $\beta>1$ ). We have checked that the following ansatz:

$$
V_{m}(\hat{x}, \hat{y})=W \hat{H} \cos q \hat{y} \frac{\sinh q \beta \hat{x}}{\sinh \beta},
$$

in which $q$ is a fitting parameter near unity, allowed us to reproduce the plots of Figs. 2(a)-2(c) at a better than qualitative level. In fact, for $q=1$ the relative difference between the simulation output and the proposed expression was everywhere less than $\pm 15 \%$ and the $q$ value that minimized the error was $q \simeq 0.9$. We have introduced the normalized units $\hat{x}=x / L$ and $\hat{y}=y / W($ note that $\beta \hat{x}=x / W)$. In the last equation, again $\hat{H} \propto H_{\perp} \sqrt{W / d}$, with a proportionality constant of order of unity which slightly increases when the barrier thickness $t_{j}$ decreases. Unfortunately, recalling the comments of the previous section, we cannot be more precise on this point.

Now we focus our attention on the components of the magnetic field in the barrier plane $H_{x}(x, y)=-\partial V_{m} /\left.\partial x\right|_{z=0}$ and $H_{y}(x, y)=-\partial V_{m} /\left.\partial y\right|_{z=0}\left(H_{z}\right.$ being identically null all over the barrier area). They are shown in Figs. 3(a) and 3(b), respec- 

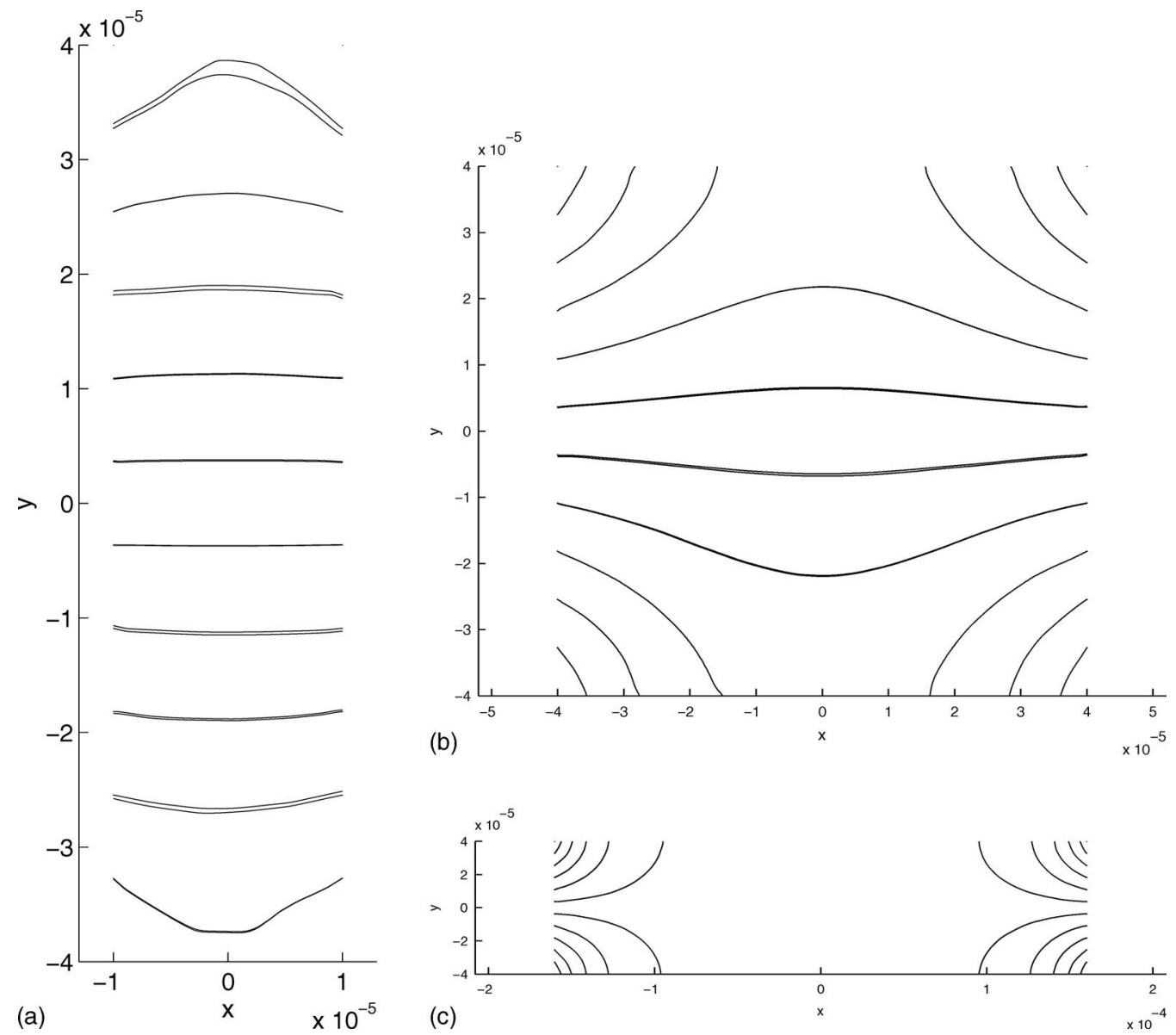

FIG. 4. Numerically obtained magnetic field streamlines inside three overlap planar Josephson tunnel junctions having the same width $2 W=80 \mu \mathrm{m}$, but different lengths: (a) $2 L=20 \mu \mathrm{m}(\beta=0.25)$, (b) $2 L=80 \mu \mathrm{m}(\beta=1)$, and (c) $2 L=320 \mu \mathrm{m}(\beta=4)$.

tively, for the particular case $W=L=100 \mu \mathrm{m}$. We would like to specify, at this point, that the same plots obtained from numerical simulations based on the vector, rather than scalar, potential differed by no more than $\pm 10 \%$, the discrepancy being larger at the barrier edges. From Eq. (6) with $q=1$, the following analytical expressions are derived:

$$
\begin{aligned}
& H_{x}(\hat{x}, \hat{y})=-\hat{H} \cos \hat{y} \frac{\cosh \beta \hat{x}}{\sinh \beta}, \\
& H_{y}(\hat{x}, \hat{y})=\hat{H} \sin \hat{y} \frac{\sinh \beta \hat{x}}{\sinh \beta} .
\end{aligned}
$$

The physical meaning of the last expressions is that for $\beta$ $=L / W>1$, the magnetic field lines are confined to the corners of the junctions at a distance $W$, and most of the field lines entering the junction at $x= \pm L$ are bent by $90^{\circ}$ and leave at $y= \pm W$. In the opposite limit, $\cosh \beta x \approx 1$, so the $x$-dependence of $H_{x}$ disappears, meaning that all the field lines entering the barrier at, say, $x=-L$, exit at $x=L$ (or vice versa). Further, we notice that while the $x$-component is negative all over the barrier area, the $y$-component symmetrically spans from negative to positive values. Due to the linearity of Eq. (4) and to the system symmetry with respect to the $z=0$ plane, if the direction of the transverse field is reverted, then $H_{x}$ and $H_{y}$ simply invert their sign. The magnetic field line distributions in the junction barrier corresponding to the scalar potentials of Figs. 2(a)-2(c) are shown in Figs. 4(a)-4(c), respectively. Similar plots based on the last analytical expressions would be practically undistinguishable at the picture resolution level; therefore, they will not be shown. From the magnetic field distributions we expect that for a given junction area $L \times W$, the critical current $I_{c}$ of a planar JTJ with pure overlap geometry $(L<W)$ modulates much faster than that of a sample with pure in-line geometry $(L>W)$. At first sight, it might seem that the effect of a transverse field is qualitatively similar to that of an in-plane field applied along the film direction, i.e., along the $x$-axis, in our case. However, this is not true at a quantitative level because, in general, $H_{x}(x, \pm W)$ is not constant in a transverse field.

Inserting Eq. (6) into Eq. (5), we derive an approximate analytical expression for the Josephson phase profile:

$$
\phi(\hat{x}, \hat{y})=h \sin \hat{y} \frac{\cosh \beta \hat{x}}{\sinh \beta},
$$

where $h=2 \pi d_{e} W \mu_{0} \hat{H} / \Phi_{0}$ is a dimensionless parameter proportional to the applied transverse field amplitude $H_{\perp}$ through $\hat{H}$. It is easy to verify that the last expression, in which we have omitted an integration constant $\phi_{0}$, satisfies Eq. (3).

With $\phi$ an odd function of $\hat{y}$, then $\langle\sin \phi\rangle=0$; therefore, the magnetic pattern $I_{c}(h)$ reduces to 
(a)
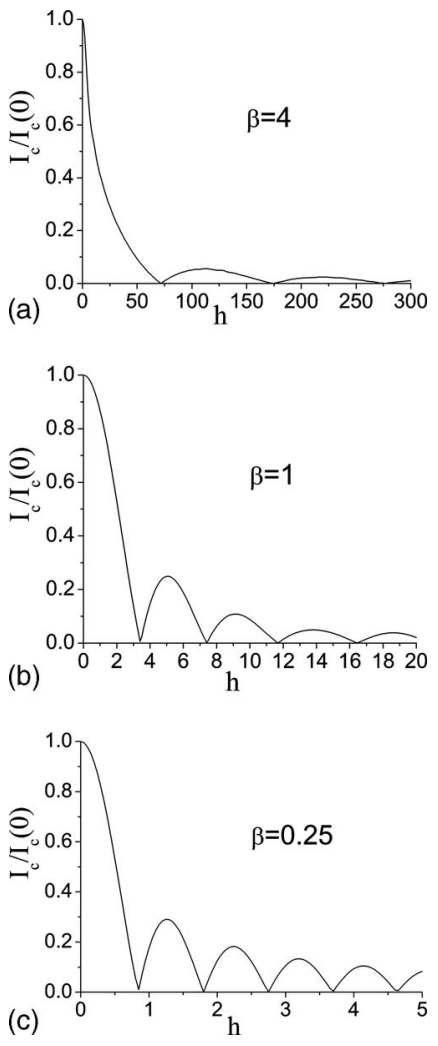

FIG. 5. Computed transverse magnetic patterns $I_{c}(h)$ for an overlap junction with different $L / W$ ratios: (a) inline junction $L=4 W$, (b) square overlap $L$ $=W$, and (c) pure overlap junction $W=4 L$.

$$
I_{c}(h)=I_{c}(0) \int_{0}^{1} d \hat{x} \int_{0}^{1} d \hat{y} \cos \phi(x, y) .
$$

Figures 5(a)-5(c) show the computed transverse magnetic patterns for the three values of the barrier aspect ratio $\beta$ used before $(4,1$, and 0.25$)$. As expected, the response to a transverse field is very weak for an in-line JTJ, the first minimum occurring at $h \simeq 74$ for $\beta=4$. The secondary pattern maxima become more pronounced for a pure overlap geometry. However, in the limit $L<<W$, all the above equations lose their validity when the overlapping length becomes comparable with the film thickness.

It is important to stress here that we are dealing with electrically small JTJs; therefore, the different shape of the transverse magnetic patterns is a direct consequence of the different distribution of the surface screening currents (and not of the applied bias current). Unfortunately, there are no data available in the literature to check the validity of our theoretical magnetic diffraction patterns for a small overlap JTJ formed by films having the same widths. In fact, the experiments reported by Rosestein and Chen in 1975 (Ref. 2) refer to an overlap JTJ formed by two thick $\mathrm{Pb}$ electrodes of unequal widths $\left(2 W=0.74 \mathrm{~mm}\right.$ and $\left.2 W^{\prime}=1.00 \mathrm{~mm}\right)$ and a common overlay region of $2 L=0.35 \mathrm{~mm}$. It is quite evident that for such geometrical film configuration, the symmetry with respect to the $y$-axis is broken and Eq. (9) is unable to correctly describe the magnetic field (and screening currents) distribution. Figures 6(a) and 6(b) show, respectively, the result of numerical simulations carried out for the specific electrode configuration of Ref. 2 and the corresponding $I_{c}(h)$.
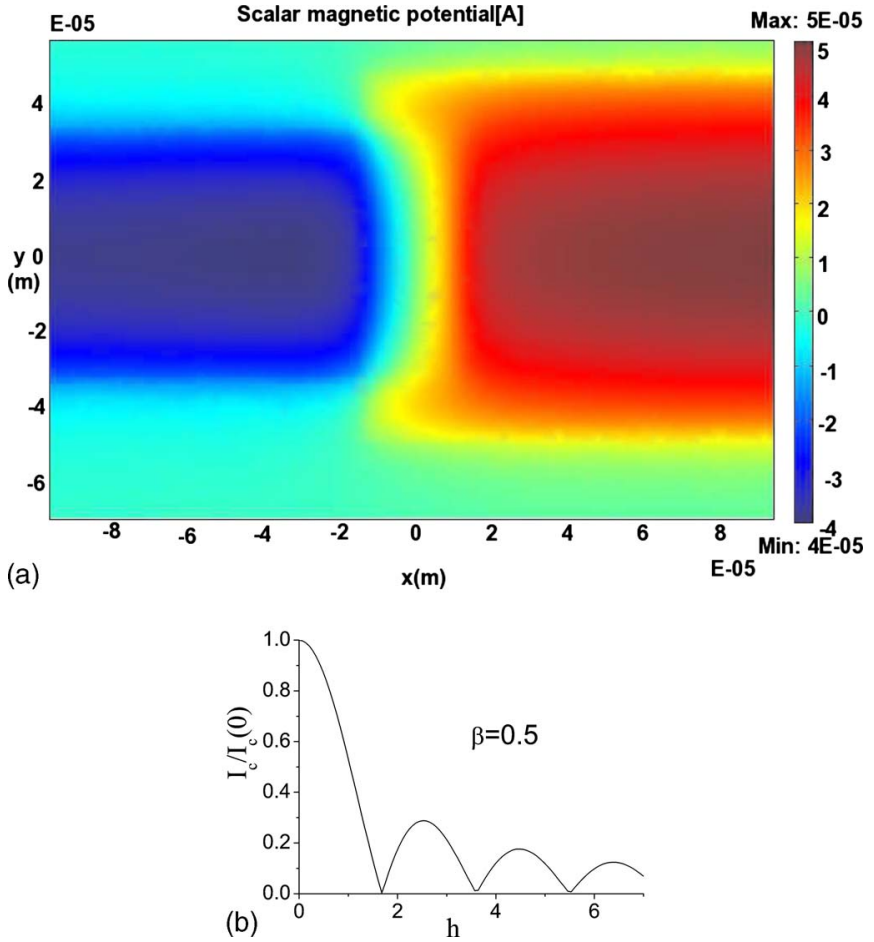

FIG. 6. (Color online) (a) Numerically obtained scalar potential for an overlap junction obtained by the superposition of two films of unequal widths $2 W=0.74 \mathrm{~mm}$ and $2 W^{\prime}=1.00 \mathrm{~mm}$. The overlapping distance is $2 L$ $=0.35 \mathrm{~mm}$, as for the sample quoted in Ref. 2. (b) Computed magnetic pattern $I_{c}(h)$ for an overlap junction with aspect ratio $L / W=0.5$.

According to Ref. 3 we believe that difference between the experimental data of Ref. 2 and the numerical prediction of Ref. 3 (valid only for the specific case $\beta=0.5$ ) arises from the unequal widths of the films in the experiment. Indeed, the magnetic diffraction pattern reported in Ref. 3 is of a piece with the curve in Fig. 6(b).

We conclude this section considering that for unidimensional overlap junctions for which $W>\lambda_{j}>L$, being $\left(\partial^{2} \phi / \partial x^{2}\right)<<\left(\partial^{2} \phi / \partial y^{2}\right)$, then the Josephson phase has to obey to the equation first introduced by Owen and Scalapino ${ }^{10}$

$$
\frac{d^{2} \phi}{d y^{2}}=\frac{1}{\lambda_{j}^{2}} \sin \phi,
$$

when an in-plane external field is applied along the $x$-direction. In fact, Fig. 7(a) shows the comparison between the diffraction patterns measured in a parallel and transverse field of a $\mathrm{Nb} / \mathrm{Al}_{\mathrm{ox}} / \mathrm{Nb}$ overlap-type junction with $\lambda_{J}$ $\approx 50 \mu \mathrm{m}$ whose length is $500 \mu \mathrm{m}$, while the width is equal to $4 \mu \mathrm{m}$. The base and top electrode widths are 540 and $506 \mu \mathrm{m}$, respectively. The junction geometry is depicted in Fig. 7(b). We observe that the two experimental data sets almost overlap, when a factor scale of about 40 is applied on the abscissa, meaning that the sample is much more sensitive to a transverse field rather than an in-plane one.

\section{B. Cross-type junctions}

Cross-geometry JTJs are formed by the superposition of two perpendicular superconducting electrodes, as depicted in Fig. 8. The static properties of such junctions in a transverse 

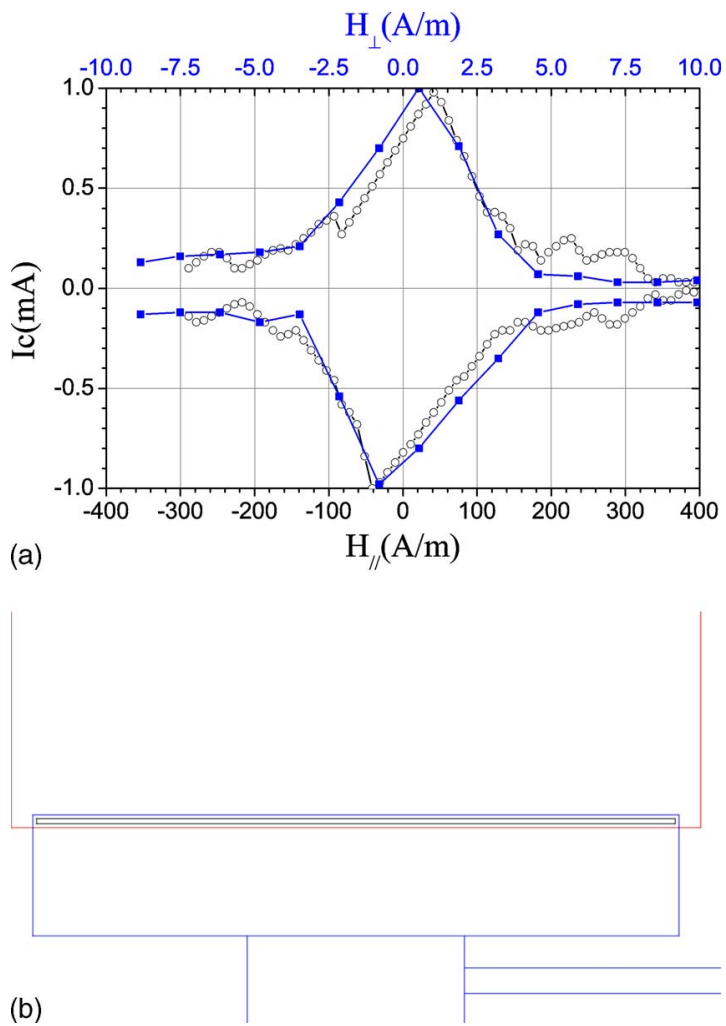

FIG. 7. (Color online) (a) Comparison between the diffraction patterns measured in a parallel (black bottom axis) and transverse (blue top axis) field of a $\mathrm{Nb} / \mathrm{Al}_{\mathrm{ox}} / \mathrm{Nb}$ overlap-type junction with $\lambda_{J}-50 \mu \mathrm{m}$ whose length is $500 \mu \mathrm{m}$, while the width is equal to $4 \mu \mathrm{m}$. (b) Geometry details: the base (red) and top (blue) electrode widths are 540 and $506 \mu \mathrm{m}$, respectively. The barrier area is delimited by the black rectangle.

magnetic field were analyzed by Miller et al. ${ }^{4}$ but only in the particular case of equal film widths $2 L=2 W$. They proposed, as an approximate solution of Eq. (3), a phase profile $\phi(x, y) \propto x y$, corresponding to a saddle-shaped scalar magnetic potential $V_{m}(x, y) \propto x^{2}-y^{2}$ and to a monotonically decreasing $I_{c}\left(H_{\perp}\right)$. We want to generalize these results for junc-

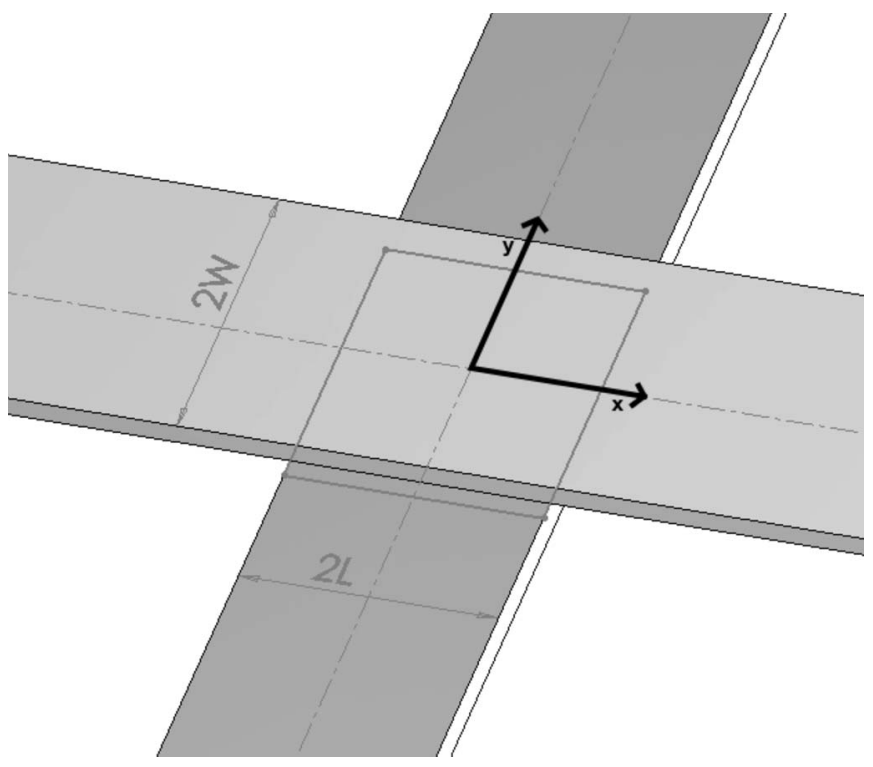

FIG. 8. Sketch of a square cross-type junction. The center of the junction coincides with the axis origin.
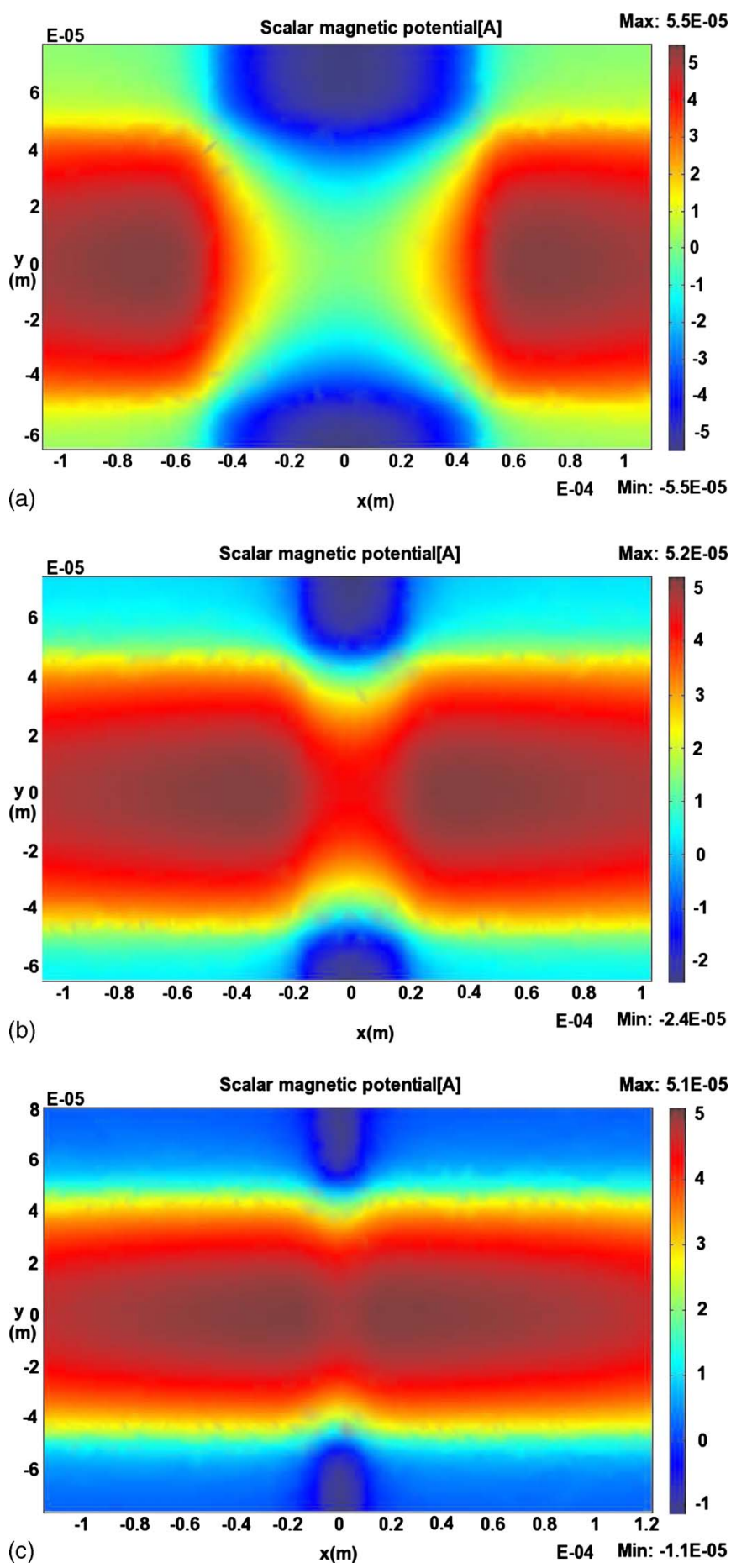

FIG. 9. (Color online) Numerically obtained magnetic scalar potential $V_{m}$ (in A) in the barrier plane for three cross-type junctions having the same width $2 W=100 \mu \mathrm{m}$, but different lengths: (a) $2 L=100 \mu \mathrm{m}(\beta=1)$, (b) $2 L$ $=40 \mu \mathrm{m}(\beta=0.4)$, and (c) $2 L=20 \mu \mathrm{m}(\beta=0.2)$. The transverse externally applied magnetic field is $H_{\perp}=1 \mathrm{~A} / \mathrm{m}$.

tions with nonunitary aspect ratios $\beta=L / W$. Figures 9(a)-9(c) display the numerical solutions of Eq. (4) for three cross-junctions having the same width, $2 W=100 \mu \mathrm{m}$, but different lengths, $2 L=100,40$, and $20 \mu \mathrm{m}$. We observe that for any value of $\beta$, the scalar potential in the barrier is fourfold symmetric, meaning that the empirical expression $V_{m}(x, y)$ we are looking for has to be an a even function of both $x$ and $y$. Further, $V_{m}$ always vanishes at the junction 
corners and, as the junction length shrinks, the scalar potential distribution gets more and more uniform over the barrier area. A careful analysis of the scalar potential plots in Figs. 9(a)-9(c) led us to the following expression:

$$
V_{m}(\hat{x}, \hat{y})=\hat{H} \sqrt{W L}\left(\cos q \hat{y} \frac{\cosh q \beta \hat{x}}{\cosh \beta}-\cos q \hat{x} \frac{\cosh q \hat{y} / \beta}{\cosh 1 / \beta}\right),
$$

in terms of normalized variables. Here again, $q$ is a fitting parameter that can be comfortably set equal to unity. More specifically, with $q=1$, the relative difference between the simulation output and the heuristic expression of Eq. (12) was numerically found to be everywhere less than $\pm 15 \%$, although it was minimized by $q \simeq 1.2$. The proposed expression is made up by two terms which can be seen as the contributions from the two electrodes. When $L=W$, the two terms have the same weights $(\operatorname{sechl} \simeq 0.65)$, but, for $\beta<1$, for example, the weight of the first term is larger than that of the second term and vice versa. Further, in the limit $\beta<$ $<1$ the first weight saturates to unity while the second vanishes.

From Eq. (12) with unitary $q$, the Josephson phase profile can be easily derived:

$$
\phi(\hat{x}, \hat{y})=h\left(\sin \hat{y} \frac{\sinh \beta \hat{x}}{\cosh \beta}+\sin \hat{x} \frac{\sinh \hat{y} / \beta}{\cosh 1 / \beta}\right),
$$

where $h=2 \pi d_{e} \sqrt{W L} \mu_{0} \hat{H} / \Phi_{0}$, and with Eq. (3) being identically satisfied. We begin with the observation that by setting $\beta=1$ and retaining the first two terms in the Taylor expansion of the trigonometric and hyperbolic functions, Eqs. (13) and (12) reduce, respectively, to $\phi(x, y) \propto x y-x^{3} y^{3} / 36$ and $V_{m}(x, y) \propto x^{2}-y^{2}$, as it should be. ${ }^{4}$ Further, upon the inversion of $\beta, \phi(\hat{x}, \hat{y})=\phi(\hat{y}, \hat{x})$, meaning that the solutions for two junctions having reciprocal aspect ratios differ by a rotation of $\pm 90^{\circ}$. Figures 10 (a) -10 (c) show the magnetic field distributions in the barrier area corresponding to the scalar potentials shown in Figs. 9(a)-9(c), respectively.

Again, with $\langle\sin \phi\rangle=0$, the magnetic diffraction pattern for a cross junction in a transverse magnetic field are found upon inserting the expression above in Eq. (10). Figure 11 shows $I_{c}(h)$ for the three values of the barrier aspect ratios considered in Figs. 9(a) -9 (c), i.e., $\beta=1,0.4$, and 0.2. For the considerations above, the red and black curves in Fig. 11 also represent the $I_{c}(h)$ for $\beta=2.5$ and 5 , respectively. We come to the interesting result that for cross junctions in a transverse field, the critical current decreases monotonically with the field amplitude $H_{\perp}$ and, for large fields $(h>>1), I_{c}(h)$ $\propto 1 / H_{\perp}$ (see the log-log plot in the inset of Fig. 11). The experimental transverse pattern presented in Ref. 4 bears strong resemblance to the $I_{c}(h)$ obtained for $\beta=1$.

\section{ANNULAR JUNCTIONS}

In this section we will examine the behavior of small annular JTJs in the presence of a transverse magnetic field. Denoting the inner and outer ring radii, respectively, as $r_{i}$ and
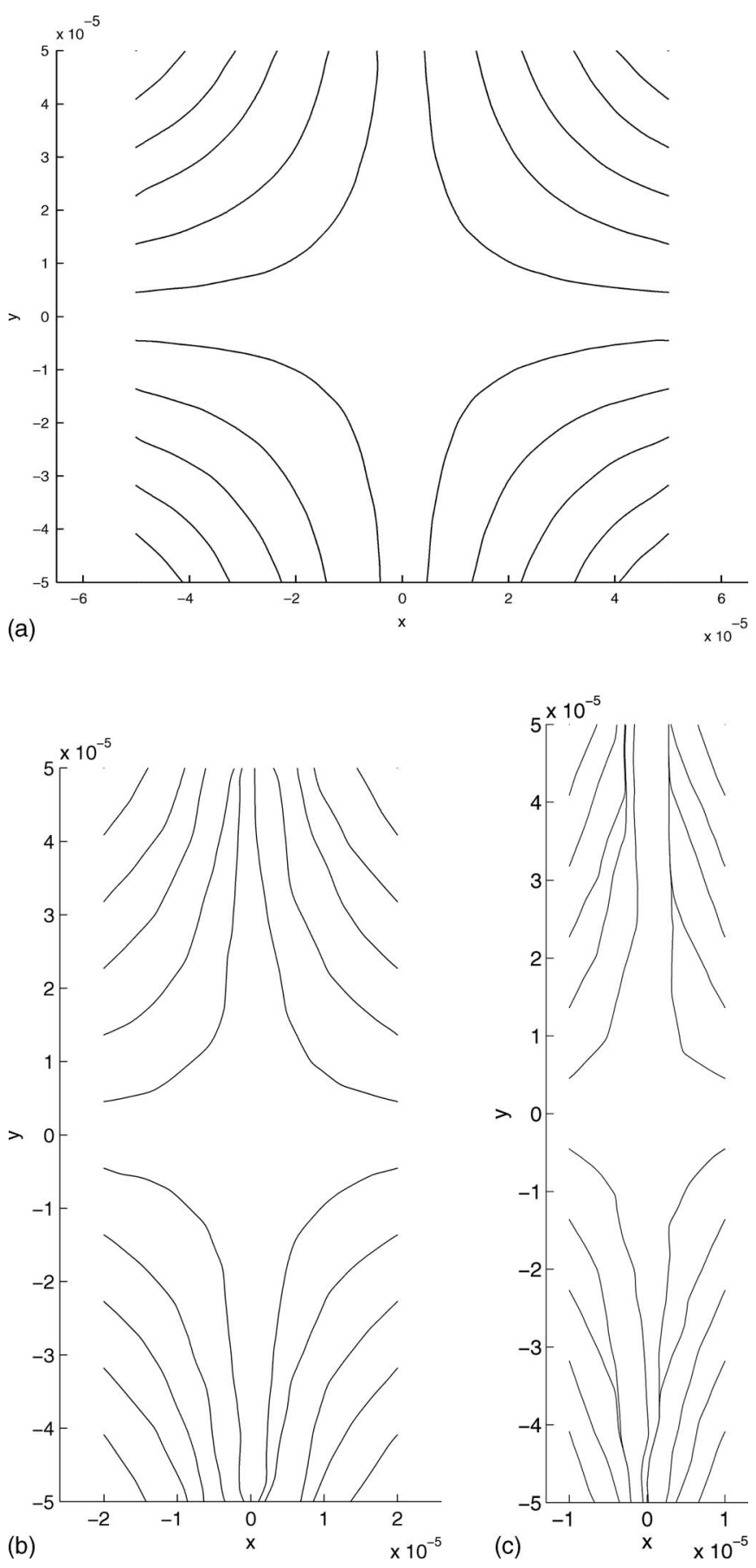

FIG. 10. Numerically obtained magnetic field streamlines inside three crosstype planar Josephson tunnel junctions having the same width $2 W$ $=100 \mu \mathrm{m}$, but different lengths: (a) $2 L=100 \mu \mathrm{m}(\beta=1)$, (b) $2 L=40 \mu \mathrm{m}$ $(\beta=0.4)$, and (c) $2 L=20 \mu \mathrm{m}(\beta=0.2)$.

$r_{o}$, we assume that the annular junction is unidimensional, i.e., the ring mean radius $\bar{r}=\left(r_{i}+r_{o}\right) / 2$ is much larger than the ring width $\Delta r=r_{o}-r_{i}$.

Using polar coordinates, the Josephson equation, Eq. (1), can be split into

$$
\frac{\partial \phi}{\partial r}=\kappa H_{\theta},
$$




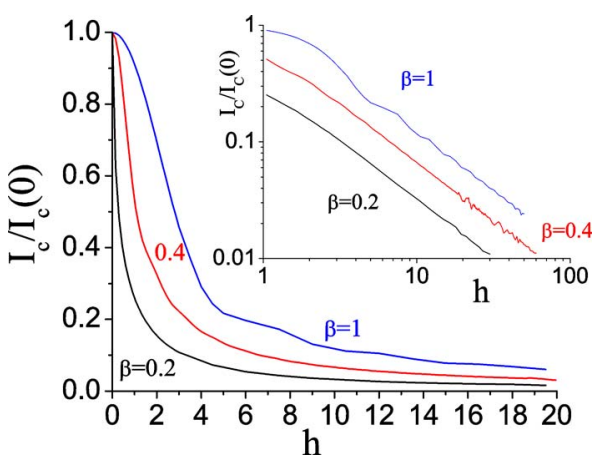

FIG. 11. (Color online) Computed transverse magnetic patterns $I_{c}(h)$ for a cross junction with different $L / W$ ratios $\beta=1, \beta=0.4$, and $\beta=0.2$. In the inset the log-log plot shows that for large fields $I_{c}(h) \propto 1 / h$.

$$
\frac{\partial \phi}{r \partial \theta}=-\kappa H_{r}
$$

where $H_{r}$ and $H_{\theta}$ are the radial and tangential components of the magnetic field in the ring plane, respectively, and $\kappa$ depends on the electrode's geometrical configuration. ${ }^{11}$ With the annulus unidimensional, we can neglect the radial dependence of the Josephson phase $\phi(r, \theta)=\phi(\bar{r}, \theta)$, and henceforth,

$$
\phi=\phi(\theta)=-\kappa \bar{r} \int d \theta H_{r}(\bar{r}, \theta)+\text { const. }
$$

In the well-known case of a spatially homogeneous in-plane field $\mathbf{H}_{\|}$applied in the direction of $\theta=0$, then $H_{r}=H_{\|} \cos \theta$ (and $H_{\theta}=H_{\|} \sin \theta$ ), so that the last integral yields ${ }^{12,13}$

$$
\phi(\theta)=h \sin \theta+\phi_{0},
$$

where $h \propto H_{\|}$and $\phi_{0}$ are integration constants. Assuming that the Josephson current density $J_{c}$ is constant over the ring circumference, the Josephson current through the barrier is obtained by

$$
I_{c}(h)=\frac{I_{c}(0)}{2 \pi} \int_{-\pi}^{\pi} d \theta \sin \phi(\theta),
$$

in which $I(0)=J_{c} 2 \pi \bar{r} \Delta r$ is the maximum junction critical current which occurs in zero field. As far as $\phi(\theta)$ is an odd function (when $\phi_{0}=0$ ), the calculation of the maximum critical current reduces to the following integration:

$$
I_{c}(h)=\frac{I_{c}(0)}{\pi} \int_{0}^{\pi} d \theta \cos \phi(\theta) .
$$

Inserting $\phi$ given by Eq. (16), we obtain for the maximum critical current, ${ }^{11}$

$$
I_{c}(h)=I_{c}(0)\left|J_{0}(h)\right|
$$

in which $J_{0}$ is the zero order Bessel function (of first kind). The periodic conditions for the phase difference $\phi$ and its angular derivative around an annular junction are

$$
\phi(\theta+2 \pi)=\phi(\theta)+2 \pi n
$$

$$
\frac{d \phi(\theta+2 \pi)}{d \theta}=\frac{d \phi(\theta)}{d \theta}
$$

where $n$ is an integer corresponding to the net number of fluxons (i.e., number of fluxons minus the number of antifluxons) trapped in the junction at the time of the normal-tosuperconducting transition. Equations (19) and (20) state that observable quantities such as the Josephson current (through $\sin \phi$ ) and the radial magnetic field (through $d \phi / d \theta$ ) must be single valued upon a round trip; they were derived in Ref. 11 starting from the fluxoid quantization.

Equations (16) and (18) hold under the assumption that there are no fluxons trapped in the barrier; however, they can be easily generalized to the case of $n \neq 0$ trapped fluxons. In such case, Eq. (16) changes to

$$
\phi(\theta)=h \sin \theta+n \theta+\phi_{0},
$$

in which the linear term $n \theta$ takes into account the phase twist due to the presence of the trapped fluxons, being that the ring circumference is smaller or comparable to the fluxon rest length. Carrying out the integration in Eq. (17) with $\phi$ given by Eq. (21) and maximizing with respect to $\phi_{0}$, we get

$$
I_{c}^{n}(h)=I_{c}(0)\left|J_{n}(h)\right|,
$$

in which $J_{n}$ is the $n$th order Bessel function. Equations (18) and (22) have been experimentally verified in a number of papers. $^{14}$

A Lyngby-type annular JTJ, first reported in 1985 by Davidson et al., ${ }^{15}$ is obtained by two films having the same width, as schematically depicted in Fig. 12(a). Further, Fig. 12(b) shows a different kind of annular JTJ for which the film widths are quite different: we will call it an asymmetric annular junction. At the end of this section we will present experimental data for such asymmetric geometrical configuration. We have carried out magnetostatic simulation for the two annular geometries depicted in Fig. 12 when the applied field is transverse. Only the case of no trapped fluxons was considered, corresponding to zero net magnetic flux through the superconducting holes. Contrary to the case of the rectangular bidimensional JTJs considered previously, now we do not have to know the magnetic field distribution in the junction plane, but by virtue of Eq. (14b), we can limit our interest to just the angular dependence of the radial magnetic field $H_{r}(\bar{r}, \theta)$. In our simulations we set $r_{i}=40 \mu \mathrm{m}$ and $r_{o}$ $=50 \mu \mathrm{m}$, so that $\bar{r}=45 \mu \mathrm{m}$. For the asymmetric configuration the film widths were chosen to be $2 W=100 \mu \mathrm{m}$ and $2 W^{\prime}=200 \mu \mathrm{m}$.

By postprocessing the simulation outputs we found out that in the case of Lyngby geometry, $H_{r}$ follows very closely a sinusoidal dependence on $\theta$, as shown in Fig. 13(a): more specifically, by choosing the angle origin in such a way that $\theta=0$ corresponds to the positive $x$-axis direction, we have $H_{r} \propto \cos \theta$, exactly as if the magnetic field were applied in the ring plane. By integration we get Eq. (21), again with $h$ depending on the geometrical film configuration and being proportional to the transverse field amplitude $H_{\perp}$. We come to the remarkable conclusion that the diffraction pattern of an electrically small annular junction with no trapped flux in a 


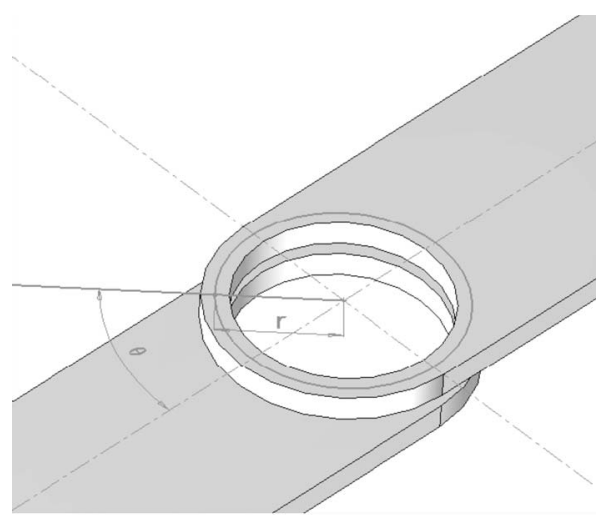

(a)

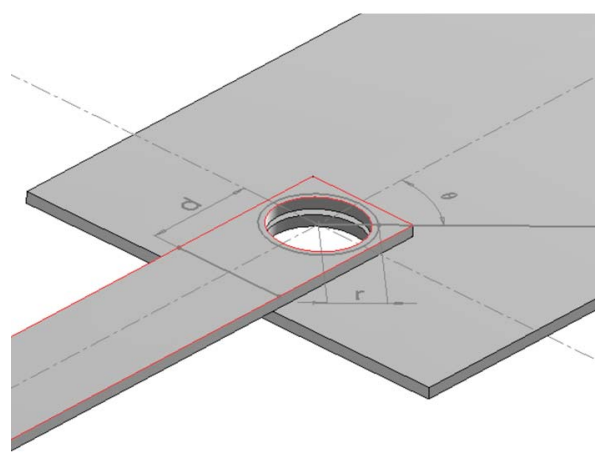

(b)

FIG. 12. (Color online) Sketches of the two types of annular JTJs considered in this paper: (a) Lyngby-type geometry made by two films whose widths match the ring diameter, and (b) asymmetric annular junctions made by two films with unequal widths.

transverse field follows the zero-order Bessel function behavior, as if the field were applied in the barrier plane.

The situation is quite different when we consider asymmetric annular junctions. In fact, as shown in Fig. 13(b), it is quite evident that now the slope of the radial field changes abruptly for $\theta \simeq \pi / 2$ and $\theta \simeq 3 \pi / 2$, resulting in a periodic asymmetric ratchetlike potential $d H_{r} / d \theta$. We have numerically checked that to a high accuracy $\int_{0}^{2 \pi} d \theta H_{r}(\theta)=0$, as it should be when no fluxons are trapped in the junction. In order to correctly reproduce $H_{r}(\theta)$, we have to consider higher $\theta$ harmonics. It was found that a truncated Fourier expansion cast in the form

$$
H_{r}(\theta) \propto \cos \theta+2 \gamma \cos 2 \theta+3 \delta \cos 3 \theta
$$

can satisfactorily fit our numerical findings. The two fitting parameters $\gamma$ and $\delta$ can be ascribed to two degrees of freedom in the layout geometry: the ratio of the top and bottom film widths and the distance from the junction to the edge of the bottom film. Equation (23) with $\gamma=0.11$ and $\delta=0.085$ is shown as a solid red line in Fig. 13(b).

By integrating Eq. (14b) with $H_{r}$ given by Eq. (23), we get an approximate expression for the angular phase dependence,

$$
\phi(\theta) \approx h(\sin \theta+\gamma \sin 2 \theta+\delta \sin 3 \theta),
$$

in which still $\phi(-\theta)=-\phi(\theta)$, but the symmetries $\phi(\pi / 2$ $-\theta)=\phi(\pi / 2+\theta)$ and $\phi(3 \pi / 2-\theta)=\phi(3 \pi / 2+\theta)$ are now lost. Since $\phi(\theta)$ is an odd function, Eq. (17) again allows us to
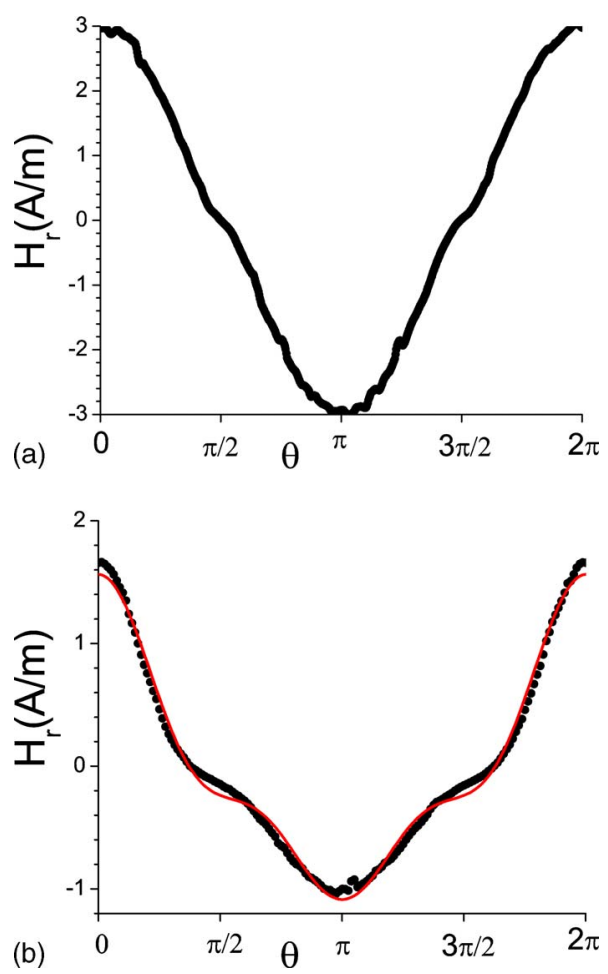

FIG. 13. (Color online) Angular dependencies of the radial magnetic field $H_{r}(\theta)$ for a small annular junction in a transverse field having (a) Lyngbytype geometry sketched in Fig. 12(a), and (b) asymmetric geometry sketched in Fig. 12(b). In the numerical simulations the amplitude of the transverse field was set to $1 \mathrm{~A} / \mathrm{m}$.

calculate the magnetic diffraction patterns corresponding to the above no-sinusoidal phase profile, even in the case when a linear term $n \theta$ is added to account for the presence of $n$ trapped fluxons. It turned out that while for $n=0$ the effects of the $\gamma$ and $\delta$ terms tend to cancel each other, resulting in a zero-order Bessel function behavior as in Eq. (18), for $n$ $\neq 0$ we found a marked departure from the $n$th order Bessel function dependence of Eq. (22). These results are supported by experimental results for an asymmetric annular junction $(\bar{r}=80 \mu \mathrm{m}$ and $\Delta r=4 \mu \mathrm{m})$ made by unequal width films: the base electrode width is $540 \mu \mathrm{m}$ and the top electrode width is $170 \mu \mathrm{m}$. For such a layout, the numerical analysis of the angular radial field dependence yielded the best fit values $\gamma=0.19$ and $\delta=0.078$. In Figs. 14(a) and 14(b) we show, respectively, the experimental diffraction patterns (dots) for such junction without trapped fluxons and with $n$ $=1$ trapped fluxon. The experimental data can be fitted very nicely by the theoretical expectations (solid red lines) obtained inserting the above $\gamma$ and $\delta$ values in Eq. (24).

We observe that when no fluxons are trapped in the asymmetric annular junctions, the transverse pattern is definitely symmetric with respect to the inversion of field direction and is barely distinguishable from the pure Bessel one; furthermore, we stress that the same sample measured with an in-plane field applied in the $\theta=0$ direction showed again a Bessel-like pattern, but the response to the applied field was about 25 times weaker.

On the contrary, with $n=1$ the transverse magnetic diffraction pattern loses its symmetry with respect to the field amplitude, i.e., $I_{c}^{1}(-h) \neq I_{c}^{1}(h)$. Furthermore, both in the ex- 

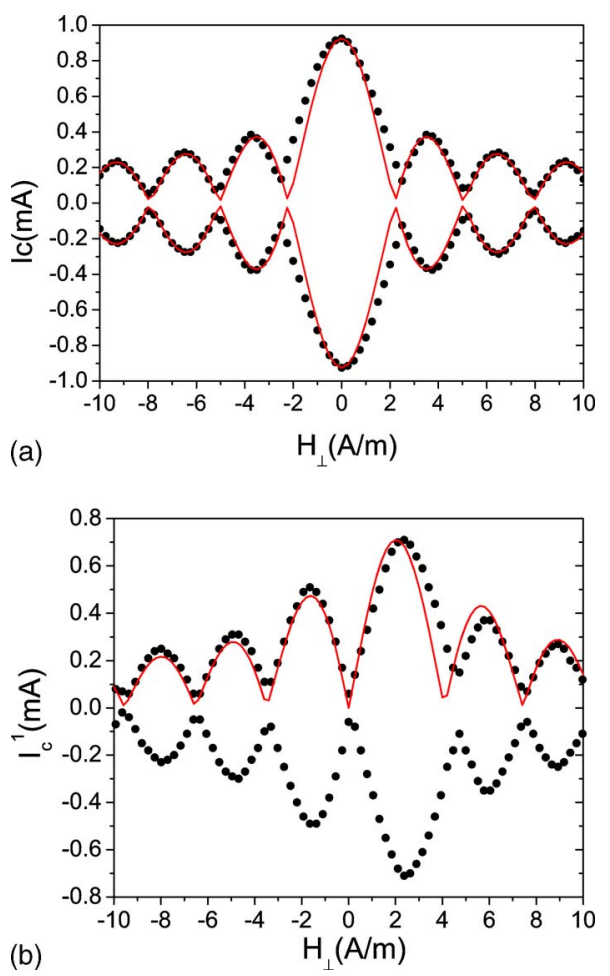

FIG. 14. (Color online) Transverse diffraction patterns for an asymmetric annular junction. The dots are the experimental data, while the solid red lines are the theoretical expectations obtained inserting $\gamma=0.18$ and $\delta$ $=0.08$ in Eq. (24). (a) No trapped fluxon and (b) one trapped fluxon.

periments and in the calculations, it turns out that $I_{c}^{1}(-h)$ $=I_{c}^{-1}(h)$; in other words, if we invert both the field and fluxon polarities we obtain the same magnetic diffraction pattern. This result was obtained and exploited in the context of a detailed investigation of the symmetry breaking during fast normal-to-superconducting phase transitions of annular JTJs recently published. ${ }^{16}$ Among other things, it has been experimentally and theoretically demonstrated that when a small transverse field is applied to the ring during the thermal quench, the probability to trap a Josephson fluxon can be very close to unity, the fluxon polarity depending on the field polarity. The ability to easily discriminate between a fluxon and an antifluxon can be conveniently exploited in the recently proposed Josephson-vortex qubits experiments with ring and heart-shaped JTJs. ${ }^{17}$ The asymmetry of the magnetic diffraction pattern can be very simply ascribed to the ratchetlike potential whose effect on the fluxon dynamic properties has been fully investigated recently. ${ }^{18}$

We conclude this section by remarking that the angular dependence of both the radial and tangential magnetic field components in the barrier of a annular JTJ do not change if the circular hole is removed from one of the electrodes. This is supported by both numerical simulations and experimental data. ${ }^{19}$ Indeed, when the ring shaped barrier is formed between a holed film and a singly connected one, the Josephson fluxon polarity is univocally related to the polarity of the quantized flux threading the hole.

\section{CONCLUDING REMARKS}

The transverse magnetic patterns of electrically small Josephson tunnel junctions have been derived numerically by solving the magnetostatic problem for different geometrical configurations of the junction electrodes and of the barrier. More specifically, from the numerical analysis of the magnetic scalar potential produced in the barrier plane by the demagnetizing currents circulating on the electrode surfaces we derived approximate and simple expressions for the Josephson phase distribution in the barrier area, which, in turn, permitted calculation of the junction critical current. Such calculations show, among other things, that for rectangular barriers the modulation of the maximum critical current never follows the Fraunhofer behavior typical of a field applied in the barrier plane; further, $I_{c}\left(H_{\perp}\right)$ strongly depends on the barrier aspect ratio $L / W$. On the contrary, the critical current modulation in a transverse field of annular JTJs without trapped fluxons is fairly close to the one corresponding to a parallel field, although in the former case, the pattern periodicity is several times smaller. When the film configuration of the annular junction is asymmetric, then the static properties depend on the polarity of the transverse field and of the trapped fluxons. It is worthy to mention that our calculations were carried out assuming that the junctions were not biased. However, in order to measure the magnetic diffraction patterns one needs to supply a transport current by an external source. As far as the JTJ is electrically small, as in cases considered in this paper, the effect of a nonuniform current distribution through the barrier is negligible. ${ }^{1}$ Nevertheless, to exclude flux from the electrode's interiors, a self-field that wraps around the films is generated ${ }^{20}$ whose effect on the Josephson phase distribution is largest when the current is largest. This situation typically occurs when the applied field is small (or absent), regardless of its orientation with respect to the barrier plane. As the external field amplitude grows, the relative effect of bias-induced screening currents decreases, and disappears when the field amplitude is such that the critical current is zero.

It is important to stress that the static properties of a small JTJ in a transverse field is strictly related to the film layout. In the case of junctions formed in a windows between two films which completely overlap each other near the junction itself, the circulating currents on the film interior surfaces are symmetric with respect to the barrier plane and result in a zero magnetic field; consequently, such JTJs will remain totally insensitive to a transverse field. This holds for overlap-type and annular geometry JTJs shown, respectively, in Fig. 1 and Fig. 12(a) when one of the electrodes is rotated by $180^{\circ}$. As mentioned in the Introduction, we also expect a very small sensitivity to a transverse field when the barrier window is located well inside the superconducting electrodes. The possibility to design multijunction chips whose each junction has its own magnetic diffraction pattern makes the physics and the application of transverse field very attractive and promising. Unfortunately, so far, very few experimental works have dealt with transverse field because of lack of theoretical understanding. We believe that this paper will stimulate other groups to fill the gap.

${ }^{1}$ A. Barone and G. Paternò, Physics and Applications of the Josephson Effect (Wiley, New York, 1982).

${ }^{2}$ I. Rosenstein and J. T. Chen, Phys. Rev. Lett. 35, 303 (1975).

${ }^{3}$ A. F. Hebard and T. A. Fulton, Phys. Rev. Lett. 35, 1310 (1975). 
${ }^{4}$ S. L. Miller, R. K. Biagi, J. R. Clem, and D. K. Finnemore, Phys. Rev. 31, 2684 (1985).

${ }^{5}$ R. Monaco, M. Aaroe, J. Mygind, and V. P. Koshelets, J. Appl. Phys. 102, 093911 (2007).

${ }^{6}$ B. D. Josephson, Rev. Mod. Phys. 36, 216 (1964).

${ }^{7}$ M. Weihnacht, Phys. Status Solidi 32, K169 (1969).

${ }^{8}$ L. D. Landau and E. M. Lifshitz, Electrodynamics of Continuous Media (Addison-Wesley, Reading, 1960).

${ }^{9}$ M. Benkraouda and R. J. Clem, Phys. Rev. B 53, 5716 (1996).

${ }^{10}$ C. S. Owen and D. J. Scalapino, Phys. Rev. 164, 538 (1967).

${ }^{11}$ N. Martucciello and R. Monaco, Phys. Rev. B 53, 3471 (1996).

${ }^{12}$ N. Martucciello and R. Monaco, Phys. Rev. B 54, 9050 (1996).

${ }^{13}$ N. Grønbech-Jensen, P. S. Lomdahl, and M. R. Samuelsen, Phys. Lett. A 154, 14 (1991).

${ }^{14}$ N. Martucciello, C. Soriano, and R. Monaco, Phys. Rev. B 55, 15157
(1997); I. V. Vernik, S. Keil, N. Thyssen, T. Doderer, A. V. Ustinov, H. Kohlstedt, and R. P. Huebener, J. Appl. Phys. 81, 1335 (1997); C. Nappi, Phys. Rev. B 55, 82 (1997).

${ }^{15}$ A. Davidson, B. Dueholm, B. Kryger, and N. F. Pedersen, Phys. Rev. Lett. 55, 2059 (1985).

${ }^{16}$ R. Monaco, J. Mygind, M. Aaroe, R. J. Rivers, and V. P. Koshelets, Phys. Rev. B 77, 054509 (2008)

${ }^{17}$ J. Clarke, Nature (London) 425, 133 (2003).

${ }^{18}$ E. Goldobin, A. Sterck, and D. Koelle, Phys. Rev. E 63, 031111 (2001) (and references therein).

${ }^{19}$ N. Martucciello, J. Mygind, V. P. Koshelets, A. V. Shchukin, L. V. Filippenko, and R. Monaco, Phys. Rev. B 57, 5444 (1998).

${ }^{20}$ A. C. Rose-Innes and E. H. Rhoderick, Introduction to Superconductivity Par.8.7 (Pergamon Press, Oxford, 1969). 